\title{
Sustained Exposure to High Carbohydrate Availability Does Not Influence Iron-Regulatory Responses in Elite Endurance Athletes
}

\author{
Alannah K.A. McKay \\ The University of Western Australia, \\ Western Australian Institute of Sport, \\ and Australian Institute of Sport \\ Nicolin Tee \\ Australian Institute of Sport
}

\author{
Avish P. Sharma \\ Griffith University
}

\author{
Peter Peeling \\ The University of Western Australia \\ and Western Australian Institute of \\ Sport \\ Marijke Welveart \\ Australian Institute of Sport and \\ University of Canberra \\ Jamie Whitfield \\ Australian Catholic University
}

\author{
David B. Pyne \\ University of Canberra
}

Rachel P.L. van Swelm and Coby M. Laarakkers

Radboud University Medical Center and Hepcidinanalysis.com

\author{
Ida A. Heikura \\ Australian Institute of Sport and \\ Australian Catholic University \\ Megan L. Ross \\ Australian Institute of Sport and \\ Australian Catholic University \\ Louise M. Burke \\ Australian Institute of Sport and \\ Australian Catholic University
}

\begin{abstract}
This study implemented a 2-week high carbohydrate (CHO) diet intended to maximize $\mathrm{CHO}$ oxidation rates and examined the iron-regulatory response to a $26-\mathrm{km}$ race walking effort. Twenty international-level, male race walkers were assigned to either a novel high $\mathrm{CHO}$ diet (MAX $=10 \mathrm{~g} / \mathrm{kg}$ body mass $\mathrm{CHO}$ daily) inclusive of gut-training strategies, or a moderate $\mathrm{CHO}$ control diet $(\mathrm{CON}=6 \mathrm{~g} / \mathrm{kg}$ body mass $\mathrm{CHO}$ daily) for a 2 -week training period. The athletes completed a 26-km race walking test protocol before and after the dietary intervention. Venous blood samples were collected pre-, post-, and $3 \mathrm{hr}$ postexercise and measured for serum ferritin, interleukin-6, and hepcidin-25 concentrations. Similar decreases in serum ferritin (17-23\%) occurred postintervention in MAX and CON. At the baseline, CON had a greater postexercise increase in interleukin-6 levels after $26 \mathrm{~km}$ of walking (20.1-fold, 95\% CI [9.2, 35.7]) compared with MAX (10.2-fold, 95\% CI [3.7, 18.7]). A similar finding was evident for hepcidin levels $3 \mathrm{hr}$ postexercise (CON=10.8-fold, 95\% CI [4.8, 21.2]; MAX = 8.8-fold, 95\% CI [3.9, 16.4]). Postintervention, there were no substantial differences in the interleukin-6 response $(\mathrm{CON}=13.6$-fold, $95 \% \mathrm{CI}$ [9.2, 20.5]; MAX $=11.2$-fold, 95\% CI $[6.5,21.3])$ or hepcidin levels $(\mathrm{CON}=7.1$-fold, $95 \% \mathrm{CI}[2.1,15.4]$; $\mathrm{MAX}=6.3$-fold, 95\% CI $[1.8,14.6])$ between the dietary groups. Higher resting serum ferritin $(p=.004)$ and hotter trial ambient temperatures $(p=.014)$ were associated with greater hepcidin levels $3 \mathrm{hr}$ postexercise. Very high $\mathrm{CHO}$ diets employed by endurance athletes to increase $\mathrm{CHO}$ oxidation have little impact on iron regulation in elite athletes. It appears that variations in serum ferritin concentration and ambient temperature, rather than dietary $\mathrm{CHO}$, are associated with increased hepcidin concentrations $3 \mathrm{hr}$ postexercise.
\end{abstract}

Keywords: ferritin, hepcidin, race walk

Iron is a key micronutrient involved in multiple biological processes relevant to athletic performance, including oxygen transport/delivery and energy production at a cellular level (Beard, 2001). Despite the importance of adequate iron stores for athletes, exerciseinduced mechanisms of iron loss can result in a negative iron balance, likely contributing to the high prevalence of iron deficiency seen in athlete populations (Sim et al., 2019). One key mechanism responsible for the increased rates of iron deficiency in athlete populations is the impact of exercise on the key iron-regulatory hormone, hepcidin. This hormone regulates iron metabolism by

McKay and Peeling are with the School of Human Sciences (Exercise and Sport Science), The University of Western Australia, Crawley, Western Australia, Australia; and the Western Australian Institute of Sport, Mt Claremont, Western Australia, Australia. McKay, Tee, Welveart, Heikura, Ross, and Burke are with the Australian Institute of Sport, Bruce, Canberra, Australian Capital Territory, Australia. Pyne and Welveart are with the Research Institute for Sport and Exercise, University of Canberra, Canberra, Australian Capital Territory, Australia. Heikura, Whitfield, Ross, and Burke are with the Mary MacKillop Institute for Health Research, Australian Catholic University, Melbourne, Victoria, Australia. Sharma is with the Griffith Sports Physiology and Performance, School of Allied Health Sciences, Griffith University, Gold Coast, Queensland, Australia. Swelm and Laarakkers are with the Department of Laboratory Medicine (TML 830), Radboud University Medical Center, Nijmegen, The Netherlands; and with Hepcidinanalysis.com, GA, Nijmegen, The Netherlands. McKay (alannah.mckay@acu.edu.au) is corresponding author. 
internalizing ferroportin (Nemeth et al., 2004b), which impedes the absorption of iron from the gut and recycling of iron by macrophages. Hepcidin activity can be directly upregulated by the inflammatory cytokine interleukin-6 (IL-6; Nemeth et al., 2004a), with exercise-induced increases in hepcidin concentrations attributed to increases in IL-6 that occur postexercise. Here, hepcidin concentrations peak 3-6 hr postexercise (Peeling et al., 2009), creating a postexercise window in which iron metabolism may be impaired. However, it appears that the athlete's iron status interacts with the magnitude of this response (Peeling et al., 2014).

In addition to regulating hepcidin expression, IL-6 plays an important role in glucose homeostasis during exercise (Pedersen \& Fischer, 2007). When muscle glycogen stores are low, the IL-6 response to exercise is increased (Steensberg et al., 2001), signaling the liver to increase hepatic glucose output in an attempt to maintain blood glucose concentrations during exercise. Investigations of acute (Badenhorst et al., 2015) and chronic (McKay et al., 2019a) low carbohydrate (CHO) availability in the athlete's diet have shown clear increases in the postexercise IL-6 response compared with conditions of high $\mathrm{CHO}$ availability. However, while exercise-induced elevations are associated with increases in hepcidin postexercise, whether exacerbated IL-6 levels in periods of low $\mathrm{CHO}$ availability are associated with augmented hepcidin levels postexercise is less definitive, with differences in hepcidin levels between dietary conditions in the acute study not reaching significance (Badenhorst et al., 2015) and differences in the baseline iron status between groups potentially confounding the results in the chronic investigation (McKay et al., 2019b). Therefore, the extent to which $\mathrm{CHO}$ manipulation can influence the postexercise hepcidin response remains unclear.

The influence of $\mathrm{CHO}$ manipulation on endurance performance has been extensively studied. High $\mathrm{CHO}$ availability prior to and during exercise enhances $\mathrm{CHO}$ storage and utilization, characteristics that are deemed beneficial to performance, as $\mathrm{CHO}$ oxidation produces more adenosine triphosphate (ATP) per unit of oxygen than fat (Leverve et al., 2007). With that in mind, the highly adaptable state of the gastrointestinal tract (Cox et al., 2010) can be trained by repetitive exposure to high doses of $\mathrm{CHO}$ during exercise to increase gastric emptying rates (Cunningham et al., 1991), improve intestinal CHO absorption, and minimize negative gastrointestinal symptoms (Costa et al., 2017a). Collectively, these processes may increase $\mathrm{CHO}$ oxidation during exercise and improve endurance performance (Jeukendrup, 2017). Importantly for iron metabolism, it may be proposed that an increase in $\mathrm{CHO}$ availability during exercise will better sustain blood glucose concentrations and supply the muscle with a more stable energy source, effectively blunting the signal for hepatic glucose production, minimizing IL-6 levels, and potentially attenuating the hepcidin response to exercise. Accordingly, a dietary strategy that can maximize endogenous $\mathrm{CHO}$ stores prior to exercise and increase exogenous $\mathrm{CHO}$ oxidation during exercise may be of benefit to both iron metabolism and performance. Therefore, the aim of the current study was to implement a novel 2-week CHO-rich dietary intervention and quantify the subsequent impact on inflammatory responses and hepcidin levels relevant to iron metabolism in athletes.

\section{Methods}

\section{Participants}

Twenty international-level male race walkers were invited to attend one of two 3-week training camps in either January or May of 2018.
Table 1 Subject Characteristics of Athletes Adhering to a CON and MAX CHO Diet

\begin{tabular}{lcc}
\hline Characteristic & CON $(\boldsymbol{n}=\mathbf{1 0})$ & MAX $(\boldsymbol{n}=\mathbf{9})$ \\
\hline Age (years) & $29.5 \pm 4.6$ & $29.6 \pm 4.3$ \\
Body mass $(\mathrm{kg})$ & $69.4 \pm 8.5$ & $69.6 \pm 4.2$ \\
$\mathrm{VO}_{2} \mathrm{max}(\mathrm{ml} / \mathrm{min} / \mathrm{kg})$ & $62.1 \pm 5.3$ & $63.8 \pm 5.8$ \\
$10 \mathrm{~km}$ personal best (min:s) & $41: 11 \pm 1: 28$ & $40: 39 \pm 1: 16$ \\
$20 \mathrm{~km}$ personal best (min:s) & $84: 42 \pm 4: 31$ & $83: 28 \pm 3: 44$ \\
\hline
\end{tabular}

Note. Values are presented as mean $\pm S D$. $\mathrm{CHO}=$ carbohydrate; $\mathrm{CON}=$ moderate; $\mathrm{MAX}=$ very high; $\mathrm{VO}_{2} \max =$ maximal aerobic capacity.

The athlete characteristics are provided in Table 1. One athlete completed in both training camps, while two athletes were removed from the sample analysis due to unusually high iron stores (serum ferritin $>300 \mu \mathrm{g} / \mathrm{L}$ ). These exclusions resulted in a total of 19 data sets from 18 athletes for analysis. Prior to participation, the athletes provided informed consent. The study was approved by the ethics committee of the Australian Institute of Sport.

\section{Study Design}

In a parallel groups design, the athletes were assigned to one of two dietary interventions: (a) a novel, higher $\mathrm{CHO} \operatorname{diet}(\mathrm{MAX})$, utilizing a combination of evidence-based and theoretical approaches to increase endogenous $\mathrm{CHO}$ stores and exogenous $\mathrm{CHO}$ availability, or (b) a moderate $\mathrm{CHO}$ control diet $(\mathrm{CON})$. Dietary allocations were made based on athlete dietary preference, while matching for characteristics, such as age, body mass (BM), $\mathrm{VO}_{2}$ max, and training history. The athletes completed a race walking test protocol prior to (baseline) and following the dietary intervention (postintervention) to assess any changes in iron regulation in response to exercise (see Figure 1). In the 3 days prior to each race walking test protocol, the athletes consumed a "race preparation diet," with the intention to replicate the nutrition practices that would typically occur prior to competition. The athletes then commenced their allocated dietary intervention for 2 weeks while following a standardized training program. This program was undertaken as a group, and it included two long walks $(+25 \mathrm{~km})$, an interval track session, and 14-km highintensity session performed on an incline. The remaining sessions were selected at the athlete's discretion to accommodate current fitness levels, residual fatigue, and training goals. All training was monitored by the research team and recorded in a training diary, with the athletes on average completing approximately $13.5 \mathrm{hr}$ of training per week (including resistance training) and covering approximately $138 \mathrm{~km} /$ week, which is representative of the typical volumes performed by elite male race walkers (Burke et al., 2017, Carr et al., 2020). After the 2-week intervention period, the 3-day "race preparation diet" was repeated, which this time was in accordance with their prescribed dietary intervention goals.

\section{Dietary Intervention}

Diets were created by a panel of accredited sports dietitians to provide a mean total daily energy intake of approximately $225 \mathrm{~kJ} / \mathrm{kg}$ $\mathrm{BM}$, equivalent to a daily energy availability of approximately $40 \mathrm{kcal}$ (168 kJ/kg fat free mass). Dietary intake was highly controlled, with all meals served to the athletes in a communal living environment and intake subsequently quantified using dietary analysis software (Food Works 8 Professional program; Xyris Software Australia Pty Ltd., Kenmore Hills, Australia). The CON 
Baseline

Postintervention

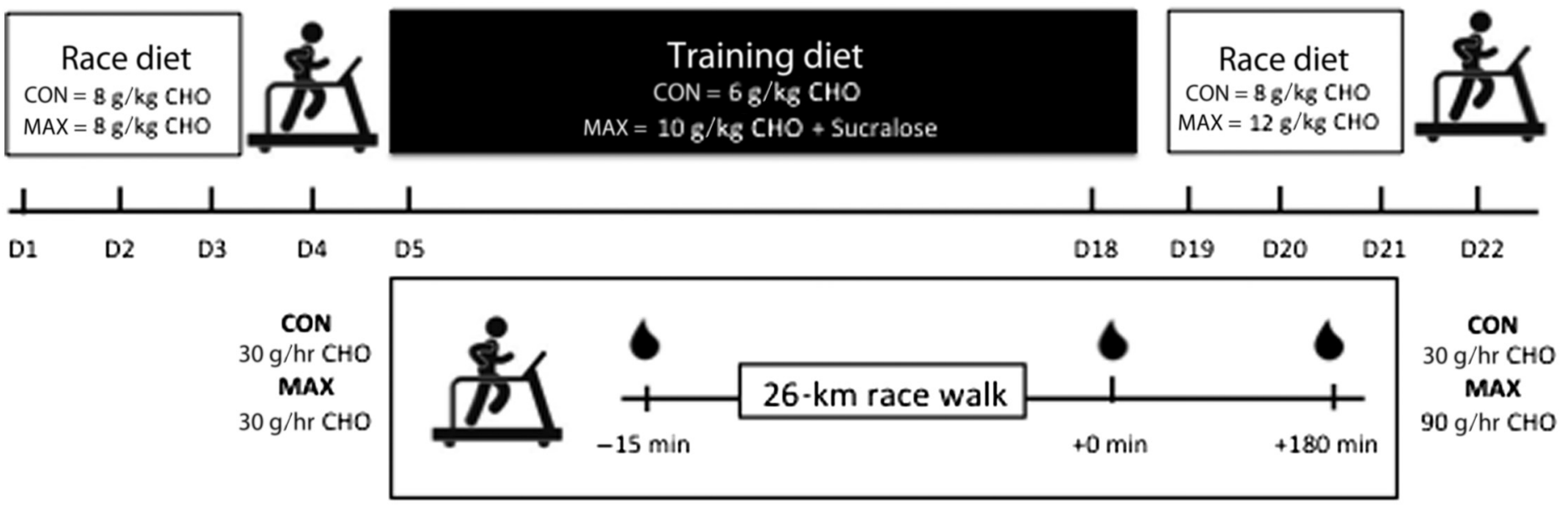

Venous blood sample

Figure 1 - Schematic representation of the study design, including CHO targets, and an overview of standardized race walking protocol. $\mathrm{CHO}=$ carbohydrate; $\mathrm{CON}=$ moderate; $\mathrm{MAX}=$ very high.

Table 2 Mean Daily Energy and Nutrient Intake of Athletes Adhering to the CON and MAX CHO Diets

\begin{tabular}{|c|c|c|c|c|c|c|}
\hline \multirow[b]{2}{*}{ Nutrient } & \multicolumn{2}{|c|}{$\begin{array}{c}\text { Baseline } \\
\text { race preparation diet }\end{array}$} & \multicolumn{2}{|c|}{ Dietary intervention } & \multicolumn{2}{|c|}{$\begin{array}{l}\text { Postintervention } \\
\text { race preparation diet }\end{array}$} \\
\hline & CON & MAX & CON & MAX & CON & MAX \\
\hline Energy (kJ/kg FFM) & $219 \pm 26$ & $225 \pm 13$ & $221 \pm 8$ & $237 \pm 13$ & $227 \pm 15$ & $245 \pm 11^{\#}$ \\
\hline Carbohydrate (g/kg BM) & $7.6 \pm 0.8$ & $8.0 \pm 0.5$ & $6.6 \pm 0.2^{*, \#}$ & $10.3 \pm 0.6^{\#}$ & $7.9 \pm 0.3^{*, * *}$ & $11.8 \pm 0.5^{\#, * *}$ \\
\hline Protein $(\mathrm{g} / \mathrm{kg} \mathrm{BM})$ & $1.8 \pm 0.3$ & $2.0 \pm 0.1$ & $2.1 \pm 0.1^{\#}$ & $2.0 \pm 0.1$ & $1.9 \pm 0.3^{*, * *}$ & $1.5 \pm 0.1^{\#, * *}$ \\
\hline Fat $(\mathrm{g} / \mathrm{kg} \mathrm{BM})$ & $1.4 \pm 0.3$ & $1.4 \pm 0.1$ & $1.8 \pm 0.1^{*, \#}$ & $0.7 \pm 0.1^{\#}$ & $1.5 \pm 0.2^{*, * *}$ & $0.6 \pm 0.1^{\#, * *}$ \\
\hline Iron $(\mathrm{mg})$ & $17.6 \pm 2.6$ & $17.8 \pm 1.5$ & $19.7 \pm 2.6^{\#}$ & $19.8 \pm 1.9^{\#}$ & $17.4 \pm 3.6$ & $19.1 \pm 1.5$ \\
\hline
\end{tabular}

Note. Values are presented as mean $\pm S D$. $\mathrm{CHO}=$ carbohydrate; $\mathrm{CON}=$ moderate; $\mathrm{MAX}=$ very high; $\mathrm{BM}=$ body mass; $\mathrm{FFM}=$ fat free mass.

*Significant difference compared with MAX. ${ }^{* *}$ Significant difference compared with the dietary intervention. ${ }^{\#}$ Significant difference compared with baseline.

diet was designed to meet the lower end of the range of recommended CHO intakes for endurance athletes (Thomas et al., 2016), providing $6 \mathrm{~g} / \mathrm{kg}$ of $\mathrm{BM} \mathrm{CHO}$ daily, with $30 \mathrm{~g} / \mathrm{hr}$ of $\mathrm{CHO}$ consumed during training sessions exceeding $60 \mathrm{~min}$ in duration. The 3-day race preparation diet increased $\mathrm{CHO}$ ingestion to $8 \mathrm{~g} / \mathrm{kg}$ of BM daily, in an attempt to maximize endogenous $\mathrm{CHO}$ stores prior to the race walking test protocol. The MAX intervention used a combination of strategies for increasing the capacity of the athlete to absorb and utilize $\mathrm{CHO}$ as a fuel source during exercise. This approach included a daily $\mathrm{CHO}$ intake $(10 \mathrm{~g} / \mathrm{kg} \mathrm{BM} \mathrm{CHO})$ at the upper end of current sports nutrition guidelines (Thomas et al., 2016). During training, emphasis was placed on athletes consuming an increasing amount of $\mathrm{CHO}$ across the 2-week intervention (from 60 up to $90 \mathrm{~g} / \mathrm{hr}$ of multiple-transportable $\mathrm{CHO}$ ). A total of $5 \mathrm{mg} / \mathrm{kg} \mathrm{BM}$ of sucralose was also consumed daily, provided as a $795 \mathrm{mg} / \mathrm{L}$ solution, with athletes instructed to drink one-third of the solution three times per day, away from any other food or fluid ingestion. Animal models have demonstrated that chronic (2-week) ingestion of sucralose can upregulate the intestinal absorption of $\mathrm{CHO}$ by increasing the activity of sodium-dependent glucose transporter (Margolskee et al., 2007). Prior to baseline testing, both the MAX and CON groups consumed the same 3-day race preparation diet. However, in the 3 days prior to postintervention testing, the MAX group's daily $\mathrm{CHO}$ intake increased to $12 \mathrm{~g} / \mathrm{kg}$ of $\mathrm{BM}$ inclusive of low-residue foods to reduce gut fiber content (Thomas et al., 2016). The dietary targets for each study phase are shown in Figure 1, with the actual dietary intakes detailed in Table 2.

Race walking test protocol. A 26-km race walking protocol was completed at baseline and postintervention to assess the impact of each dietary intervention on inflammatory and iron-regulatory responses to exercise. For each test, the athletes arrived at the laboratory at the same time each morning and consumed a standardized breakfast consisting of $2 \mathrm{~g} / \mathrm{kg}$ of BM CHO. Fifteen minutes prior to the start of exercise (105 min after breakfast), a 4-ml venous blood sample was drawn from an indwelling cannula placed into a forearm vein. The athletes then commenced a combined laboratory and field race walking protocol. Kilometers 0-1, 6-7, 12-13, 18-19, and 24-25 were performed on a motorized treadmill at either 12 or $13 \mathrm{~km} / \mathrm{hr}$. This speed equated to approximately $75 \% \quad \mathrm{VO}_{2} \mathrm{max}$ and corresponded approximately to the athlete's $50-\mathrm{km}$ race pace. The athletes then immediately commenced the final kilometer (25-26), with a speed increment of 
$1.3 \mathrm{~km} / \mathrm{hr}$ (to 13.3 or $14.3 \mathrm{~km} / \mathrm{hr}$ ). This speed increase was used to replicate the top-end finishing speed or end spurt that typically occurs during the final stages of a race walking event (Hanley, 2013). The remaining kilometers were performed on an outdoor circuit (5-km loop) at a consistent speed, nominated by the athlete, which was maintained between trials. Prior to exercise, and following each kilometer completed on the treadmill, the athletes were provided with a $\mathrm{CHO}$ solution consisting of a 2:1 glucose to fructose ratio. During baseline testing, this solution was $8 \% \mathrm{CHO}$ and provided approximately $30 \mathrm{~g} / \mathrm{hr}$. The athletes adhering to the CON diet replicated this intake during the postintervention testing; however, the athletes allocated to the MAX intervention consumed a 24\% CHO solution providing approximately $90 \mathrm{~g} / \mathrm{hr}$. Immediately postexercise, a $2.5-\mathrm{ml}$ blood sample was collected. At $30 \mathrm{~min}$ postexercise, the athletes consumed a protein drink and rested in the laboratory until $3 \mathrm{hr}$ postexercise, where a final blood sample $(2.5 \mathrm{ml})$ was collected.

\section{Venous Blood Analysis}

A total of three venous blood samples were collected during each race walking test protocol. Samples were collected into a 4-ml (preexercise) or 2.5-ml (post- and $3 \mathrm{hr}$ postexercise) serum separator tube, which were subsequently left on the bench top at room temperature for $30 \mathrm{~min}$ to clot. The samples were then centrifuged at 2,200 $\mathrm{g}, 4{ }^{\circ} \mathrm{C}$, for $10 \mathrm{~min}$. Serum iron and ferritin analyses were immediately determined on preexercise samples via a COBAS Integra 400 automated biochemistry analyzer biochemistry analyzer (Roche Diagnostics, Rotkreuz, Switzerland). The remaining serum was aliquoted and frozen at $-80^{\circ} \mathrm{C}$ until a batch analysis was conducted. Preexercise, an additional $2 \mathrm{ml} \mathrm{K} \mathrm{K}_{3}$ EDTA tube was collected and whole blood was immediately analyzed for hemoglobin and hematocrit by fluorescent flow cytometry on a XN-L 550 analyzer (Sysmex Corporation, Kobe, Japan).

Concentrations of IL-6 were analyzed pre- and postexercise using a commercially available ELISA (Quantikine HS, R\&D Systems, Minneapolis, MN) on a FLUOstar OPTIMA plate reader (BMG Labtech, Ortenberg, Germany). The coefficient of variation for IL-6 determination was $4.9 \%$. Hepcidin measurements were performed on pre- and 3-hr postexercise samples by a combination of weak cation exchange chromatography and time-of-flight mass spectrometry using synthetic hepcidin-24 as an internal standard for quantification (Kroot et al., 2010; Swinkels et al., 2008). Hepcidin-25 concentrations are expressed as nmol/L (nM). The lower limit of detection of this method is $0.5 \mathrm{nM}$, and data points below this value were substituted with the lower limit of detection divided by $\sqrt{2}$ (Croghan \& Egeghy, 2003).

\section{Statistical Analysis}

The data were analyzed with a general linear mixed model using the $\mathrm{R}$ package lme4 (R Core Team, 2017) to accommodate the unbalanced design and the repeated measurements (Jennrich \& Schluchter, 1986). A random intercept for both athlete and camp was included to adjust for the baseline levels and interindividual homogeneity, and to account for the partial cross-over design. All models were estimated using restricted maximum likelihood. Visual inspection of the residual plots did not reveal any obvious deviations from homoscedasticity or normality. The $p$ values were obtained using Type II Wald $F$ tests with Kenward-Roger degrees of freedom, as implemented in the R package car (Fox \& Weisberg, 2011). To account for differences in ambient temperature during each trial, the mean ambient temperature for each individual's trial was included as a covariate in the analysis of IL-6 and hepcidin concentrations. Furthermore, given the strong influence that iron status can have on the postexercise hepcidin response (Peeling et al., 2014), serum ferritin was used as a covariate in the analysis of hepcidin. Pre- to postexercise changes in IL-6 and hepcidin are expressed as X-fold changes. To construct a confidence interval (CI) around the fold change, a bootstrapped CI based on 10,000 replications was calculated using the R package boot (Canty \& Ripley, 2017).

\section{Results}

Serum ferritin concentrations decreased from the baseline to postintervention in both groups, $F(1,17)=5.08, p=.039$, with the magnitude of decrease similar between groups, $F(1,17)=0.726$, $p=.411$; Table 3 . No differences in serum ferritin were evident between groups at the baseline or postintervention, $F(1,10)=2.0$, $p=.189$. The serum iron concentrations were similar between groups, $F(1,15)=0.53, p=.479$, and trials, $F(1,16)=0.85, p=.371$. The hemoglobin concentrations, $F(1,17)=6.95, p=.017$, and hematocrit, $F(1,17)=4.96, p=.040$, decreased from the baseline to postintervention; however, no differences between groups were evident. In the MAX group, the ambient temperature was greater during postintervention, compared with the baseline, $F(1,17)=8.715, p=.009$; however, no difference between trials for CON or differences between dietary groups were evident. Significant differences in humidity were also evident between the dietary interventions, $F(1$, $16)=4.59, p=.048$, and trials, $F(1,17)=19.47, p=.004$.

The concentrations of IL- 6 consistently increased from pre- to postexercise, $F(1,51)=157.32, p<.001$, the magnitude of which was generally greater in the CON than the MAX group, $F(1,51)=$ $5.11, p=.03$; Figure 2a. While these differences did not reach significance between trials, $F(1,51)=1.49, p=.22$, a greater postexercise increase was evident at the baseline in CON (20.1-fold increase; 95\% CI [9.2, 35.7]), compared with MAX (10.2-fold increase; $95 \%$ CI $[3.7,18.7]$, mean estimate $=10.8 \mathrm{pg} / \mathrm{ml} ; 4.2$ to $14.6 \mathrm{pg} / \mathrm{ml})$. The equivalent comparison during the postintervention trial revealed no differences between the CON (13.6-fold increase; 95\% CI $[9.2,20.5])$ and MAX groups (11.2-fold increase; $95 \% \mathrm{CI}$ $[6.5,21.3]$, mean estimate $=-1.6 \mathrm{pg} / \mathrm{ml} ;-5.3$ to $4.9 \mathrm{pg} / \mathrm{ml})$.

An increase in hepcidin-25 concentrations was evident at $3 \mathrm{hr}$ postexercise, $F(1,51)=70.24, p<.001$; Figure $2 \mathrm{~b}$. Specifically, the increase in hepcidin-25 was greater at the baseline in CON (10.8-fold increase; $95 \%$ CI $[4.8,21.2])$ than MAX (8.8-fold increase; 95\% CI $[3.9,16.4]$; mean estimate $=8.7 \mathrm{nM} ; 4.8$ to $11.4 \mathrm{nM}$ ). However, postintervention, no differences in the hepcidin-25 increase were evident between the CON (7.1-fold increase; 95\% CI [2.1, 15.4]) and MAX group (6.3-fold increase; $95 \%$ CI $[1.8,14.6]$, mean estimate $=$ $3.6 \mathrm{nM} ;-5.4$ to $0.6 \mathrm{nM})$. Serum ferritin, $F(1,27)=9.80, p=.004$, and ambient temperature, $F(1,37)=6.74, p=.014$ were both significant variables in the hepcidin model, indicating that higher resting serum ferritin and hotter trial temperatures were associated with higher hepcidin levels $3 \mathrm{hr}$ postexercise.

\section{Discussion}

This study demonstrates that the 2-week novel MAX dietary intervention provided no additional benefits to inflammatory control or hepcidin activity following a $26-\mathrm{km}$ standardized race walking protocol, compared with a more moderate $\mathrm{CHO}$ intake. This outcome is in agreement with some of our previous work, 
Table 3 Hematological Variables and Environmental Conditions During the 26-km Race Walking Protocol for Athletes Adhering to a CON and MAX CHO Diet During the Baseline and Postintervention Trials

\begin{tabular}{|c|c|c|c|c|}
\hline & \multicolumn{2}{|c|}{ MAX } & \multicolumn{2}{|c|}{ CON } \\
\hline & Baseline & Postintervention & Baseline & Postintervention \\
\hline \multicolumn{5}{|c|}{ Serum ferritin $(\mu \mathrm{g} / \mathrm{L})$} \\
\hline Mean & 51 & $43^{* *}$ & 81 & $62^{* *}$ \\
\hline$S D$ & 27 & 22 & 33 & 34 \\
\hline \multicolumn{5}{|c|}{ Serum iron $(\mu \mathrm{mol} / \mathrm{L})$} \\
\hline Mean & 13.7 & 14.1 & 17.8 & 15.6 \\
\hline$S D$ & 2.2 & 4.2 & 6.0 & 8.2 \\
\hline \multicolumn{5}{|c|}{ Hemoglobin (g/dl) } \\
\hline Mean & 14.9 & $14.7^{* *}$ & 15.5 & $14.6^{* *}$ \\
\hline$S D$ & 0.8 & 1.0 & 1.7 & 1.0 \\
\hline \multicolumn{5}{|c|}{ Hematocrit (\%) } \\
\hline Mean & 42.9 & $42.2^{* *}$ & 44.4 & $42.4^{* *}$ \\
\hline$S D$ & 2.0 & 0.8 & 4.6 & 2.9 \\
\hline \multicolumn{5}{|c|}{ Temperature $\left({ }^{\circ} \mathrm{C}\right)$} \\
\hline Mean & 17.6 & $19.6^{* *}$ & 16.4 & 15.0 \\
\hline$S D$ & 3.9 & 1.7 & 4.4 & 5.3 \\
\hline \multicolumn{5}{|c|}{ Humidity (\%) } \\
\hline Mean & 41 & $49^{* *}$ & $52 *$ & $60^{*, * *}$ \\
\hline$S D$ & 10 & 5 & 11 & 8 \\
\hline
\end{tabular}

Note. Values are presented as mean $\pm S D$. $\mathrm{CON}=$ moderate; $\mathrm{MAX}=$ very high; $\mathrm{CHO}=$ carbohydrate.

*Significant difference compared with MAX. **Significant difference compared with baseline.

indicating that chronic macronutrient manipulations may not have a strong influence on iron regulation in elite endurance athletes (McKay et al., 2019b). Instead, it appears that resting iron status (serum ferritin concentrations) and environmental conditions (ambient temperature) had a larger influence on the increase in hepcidin levels $3 \mathrm{hr}$ postexercise than dietary $\mathrm{CHO}$ intake.

A similar decrease in iron status (determined via serum ferritin) was evident after 2 weeks in both the CON (-23\%) and MAX $(-17 \%)$ group. This short-term reduction in serum ferritin occurred, despite dietary iron intakes being well above the recommended daily intake of $8 \mathrm{mg}$ /day for males in both groups (Trumbo et al., 2001). Our findings are in-line with previous studies showing that iron stores can decrease when individuals undertake heavy periods of physical training (Auersperger et al., 2013; Karl et al., 2010), even when dietary iron intake is adequate (McKay et al., 2019a). Although this pattern may reflect a positive adaptation to the training stimulus with iron stores used to synthesize new tissues and proteins, it appears that decreases in serum ferritin can occur in as little as 2 weeks with high volume/intensified training loads. Therefore, preparation for this type of training should occur, as is the case for specialized interventions, such as altitude exposure, to ensure that the athlete's iron status is sufficient to support the desired adaptive response (Govus et al., 2015). This requirement is a key consideration for dietitians and sports physicians working with endurance athletes who might be at a higher risk of compromised iron stores.

Differences in IL-6 levels were evident between groups at baseline, but not postintervention, which might relate to the attenuated IL-6 response that occurred within the CON group postintervention. Training can attenuate IL-6 levels postexercise in both recreationally active individuals (Croft et al., 2009) and elite race walkers (McKay et al., 2019a). As a result of training, the muscle becomes less dependent on glucose and muscle glycogen as a substrate during submaximal exercise (Hennigar et al., 2017), subsequently minimizing the signal for muscle-derived IL-6 release. Meanwhile, the MAX group experienced a similar IL-6 response during both trials; that is, they did not experience the same attenuation in IL-6 postintervention as CON, despite demonstrating similar improvements in peak aerobic capacity following the dietary intervention to the CON group (unpublished data). A potential explanation is that the increased amount of $\mathrm{CHO}$ ingested by the MAX group during the postintervention trial $(90 \mathrm{~g} / \mathrm{hr})$ resulted in larger perturbations to gastrointestinal integrity and function and, combined with the hotter trial temperature, potentially signaled an increased inflammatory response to exercise (Costa et al., 2017b; Starkie et al., 2005). Nevertheless, the difference in the baseline values between groups, in combination with the high variability in IL-6, makes it difficult to isolate any meaningful impact of the dietary interventions. It seems that moderate CHO intake may be sufficient in preventing large increases in postexercise IL-6 concentrations, and there is no additional benefit (to the inflammatory response) from increasing $\mathrm{CHO}$ ingestion further.

At $3 \mathrm{hr}$ postexercise, the hepcidin response was greater in the $\mathrm{CON}$ than the MAX group at the baseline, with no difference between groups evident postintervention, again, likely related to an attenuated response in CON postintervention. While increases in hepcidin levels occurred in a similar manner to that of IL-6, our results demonstrated that higher serum ferritin concentrations were associated with greater hepcidin levels, implying that serum ferritin has a larger role in determining the magnitude of postexercise hepcidin increase. Indeed, the previous modeling of the ironregulatory response to exercise indicated that serum ferritin, rather than IL-6, has the greatest influence on hepcidin concentrations $3 \mathrm{hr}$ postexercise (Peeling et al., 2017). Furthermore, our earlier work has shown that dietary-induced changes in IL-6 can occur without 


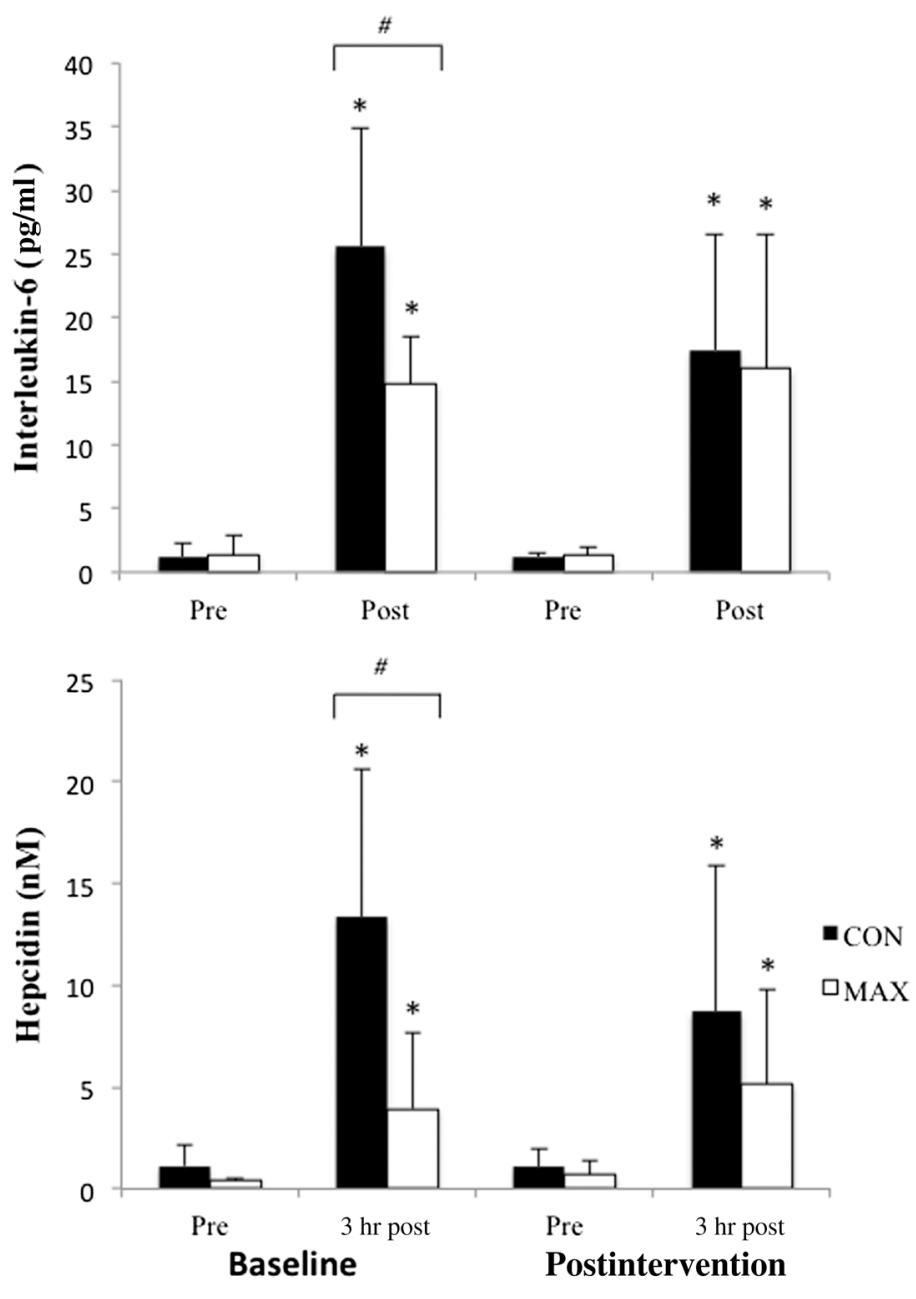

Figure 2 - (a) IL-6 concentrations pre- and postexercise and (b) hepcidin-25 concentrations pre- and $3 \mathrm{hr}$ postexercise with athletes adhering to a $\mathrm{CON}$ and $\mathrm{MAX} \mathrm{CHO}$ diet during the baseline and postintervention trials. Results presented as mean $\pm S D$. IL- $6=$ interleukin6; $\mathrm{CON}=$ moderate; $\mathrm{MAX}=$ very high; $\mathrm{CHO}=$ carbohydrate; $\mathrm{CI}=$ confidence interval. *Significant increase from preexercise. "Differences between dietary interventions based on CIs.

subsequent alterations to hepcidin concentrations (McKay et al., 2019b). Taken together, it appears that serum ferritin concentrations may be the most dominant factor in the regulation of postexercise hepcidin levels. Given the similarities between the IL-6 and hepcidin response to exercise, it is possible that IL-6, driven by the dietary intervention, also influenced the hepcidin levels. However, it is more likely that the observed changes to the hepcidin levels in the 3-hr postexercise period occurred as part of a homeostatic response to the lower serum ferritin concentrations (Peeling et al., 2014; Peeling et al., 2017).

The other variable associated with hepcidin concentrations in the current study was the ambient temperature of each trial. With daily and seasonal differences in weather occurring between the two training camps, and a large proportion of each trial conducted outdoors, we deemed it important to account for the effect of ambient temperature on the inflammatory and hepcidin response to exercise. Although we demonstrated that hotter trial temperatures were associated with a greater hepcidin response, ambient temperature was not associated with increased IL-6 concentrations. To our knowledge, only one study has examined the impact of heat on hepcidin activity; however, in this instance, heat was combined with hypoxia, and the impact of temperature alone was not determined (Hayashi et al 2020). Therefore, we are unsure of the mechanisms underpinning the association between heat and hepcidin concentrations evident in our study. It is of particular interest that this relationship appeared to occur independent of IL-6, despite the fact that exercising in the heat can augment the IL-6 response to exercise (Starkie et al., 2005), which potentially implicates a noninflammatory mechanism of upregulation. However, it should be emphasized that our findings do not infer causality; therefore, there is the possibility that a third factor, affected by both hepcidin levels and ambient temperature, has mediated this association. Nevertheless, our findings provide support for further work to be undertaken to establish the impact of an athlete's thermal environment on the hepcidin response to exercise and the subsequent impact on iron absorption.

There are inherent limitations associated with implementing applied field-based research with elite athletes that should be acknowledged. First, employing an outdoor exercise protocol meant that the differences in environmental conditions may have affected our ability to directly assess the impact of the dietary interventions. While environmental conditions were accounted for statistically, the impact that changing conditions had on factors, such as blood pressure, sweat rates, and plasma volume, was not quantified here. Furthermore, given no research has yet examined iron-regulatory responses to exercising in the heat, the full implications of the current study remain unclear. In addition, there were many elements included in the novel MAX dietary intervention, including differing $\mathrm{CHO}$ intakes, gut-training strategies, low residual diets, and the periodic ingestion of sucralose. Accordingly, the current study was unable to isolate the direct impact each of these factors had on iron metabolism, instead, assessing the practical implications to iron regulation from implementing this strategy as a whole. Finally, this study exclusively studied male athletes. With the prevalence of iron deficiency higher in females, compared with males athletes (Rowland, 2012), assessing the potential for CHO-based nutritional interventions to improve iron metabolism in females is warranted.

\section{Conclusion}

Very high CHO diets (10-12 g/kg/day), such as those practiced by endurance athletes as part of gut-training strategies, do not have a clear impact on iron regulation in elite athletes. Our data confirms previous research demonstrating that serum ferritin levels have a strong influence on postexercise hepcidin concentrations (McKay et al., 2019b; Peeling et al., 2014; Peeling et al., 2017). Moreover, ambient temperature may also be associated with the increase in hepcidin concentrations $3 \mathrm{hr}$ postexercise; this relationship warrants further investigation.

\section{Acknowledgments}

This study was financially supported by grants from the Australian Catholic University Research Fund and the Alliance for Potato Research and Education. The study was designed by A.K.A. McKay, P. Peeling, D.B. Pyne, N. Tee, I.A. Heikura, A.P. Sharma, J. Whitfield, M.L. Ross, and L.M. Burke; the data were collected and analyzed by A.K.A. McKay, N. Tee, M. Welveart, I.A. Heikura, A.P. Sharma, J. Whitfield, M.L. Ross, R.P.L. van Swelm, C.M. Laarakkers, and L.M. Burke; drafting the article or revising it critically for important intellectual content was undertaken by A.K.A. McKay, P. Peeling, D.B. Pyne, and L.M. Burke. All authors approved the final version of the paper. 


\section{References}

Auersperger, I., Skof, B., Leskosek, B., Knap, B., Jerin, A., \& Lainscak, M. (2013). Exercise-induced changes in iron status and hepcidin response in female runners. PLoS One, 8(3), e58090. PubMed ID: 23472137 doi:10.1371/journal.pone.0058090

Badenhorst, C.E., Dawson, B., Cox, G.R., Laarakkers, C.M., Swinkels, D.W., \& Peeling, P. (2015). Acute dietary carbohydrate manipulation and the subsequent inflammatory and hepcidin responses to exercise. European Journal of Applied Physiology, 115(12), 2521-2530. PubMed ID: 26335627 doi:10.1007/s00421-015-3252-3

Beard, J.L. (2001). Iron biology in immune function, muscle metabolism and neuronal functioning. Journal of Nutrition, 131(2S-2), 568S580S. PubMed ID: 11160590 doi:10.1093/jn/131.2.568S

Burke, L.M., Ross, M.L., Garvican-Lewis, L.A., Welvaert, M., Heikura, I.A., Forbes, S.G., . . Hawley, J.A. (2017). Low carbohydrate, high fat diet impairs exercise economy and negates the performance benefit from intensified training in elite race walkers. The Journal of Physiology, 595(9), 2785-2807. doi:10.1113/JP273230

Canty, A., \& Ripley, B. (2017). Package 'boot'. [Internet]. Available from http://cran.rproject.org/web/packages/boot/index.html

Carr, A.J., Saunders, P.U., Garvican-Lewis, L.A., \& Vallance, B.S. (2020). Altitude and heat training in preparation for competitions in the heat: A case study. International Journal of Sports Physiology and Performance, . 15(9), 1344-1348. doi:10.1123/ijspp.2019-0292

Costa, R.J.S., Miall, A., Khoo, A., Rauch, C., Snipe, R., Camões-Costa, V., \& Gibson, P. (2017a). Gut-training: The impact of two weeks repetitive gut-challenge during exercise on gastrointestinal status, glucose availability, fuel kinetics, and running performance. Applied Physiology, Nutrition and Metabolism, 42(5), 547-557. doi:10.1139/apnm2016-0453

Costa, R.J.S., Snipe, R.M.J., Kitic, C.M., \& Gibson, P.R. (2017b). Systematic review: Exercise-induced gastrointestinal syndrome-implications for health and intestinal disease. Alimentary Pharmacology \& Therapeutics, 46(3), 246-265. doi:10.1111/apt.14157

Cox, G.R., Clark, S.A., Cox, A.J., Halson, S.L., Hargreaves, M., Hawley, J.A., ... Burke, L.M. (2010). Daily training with high carbohydrate availability increases exogenous carbohydrate oxidation during endurance cycling. Journal of Applied Physiology, 109(1), 126134. PubMed ID: 20466803 doi:10.1152/japplphysiol.00950.2009

Croft, L., Bartlett, J.D., MacLaren, D.P., Reilly, T., Evans, L., Mattey, D.L., $\ldots$ Morton, J.P. (2009). High-intensity interval training attenuates the exercise-induced increase in plasma IL-6 in response to acute exercise. Applied Physiology, Nutrition and Metabolism, 34(6), 1098-1107. doi:10.1139/H09-117

Croghan, C., \& Egeghy, P. (2003). Methods of dealing with values below the limit of detection using SAS. Southern SAS User Group, 22, 24.

Cunningham, K.M., Horowitz, M., \& Read, N.W. (1991). The effect of short-term dietary supplementation with glucose on gastric emptying in humans. British Journal of Nutrition, 65(1), 15-19. PubMed ID: 1997128 doi:10.1079/BJN19910061

Fox, J., \& Weisberg, S. (2011). An R companion to applied regression. 2nd ed. Thousand Oaks, CA: SAGE.

Govus, A.D., Garvican-Lewis, L.A., Abbiss, C.R., Peeling, P., \& Gore, C.J. (2015). Pre-altitude serum ferritin levels and daily oral iron supplement dose mediate iron parameter and hemoglobin mass responses to altitude exposure. PLoS One, 10(8), e0135120. PubMed ID: 26263553 doi:10.1371/journal.pone.0135120

Hanley, B. (2013). An analysis of pacing profiles of world-class racewalkers. International Journal of Sports Physiology and Performance, 8(4), 435-441. PubMed ID: 23237850 doi:10.1123/ijspp.8.4.435
Hayashi, N., Yatsutani, H., Mori, H., Ito, H., Badenhorst, C.E., Goto, K., (2020). No effect of supplemented heat stress during an acute endurance exercise session in hypoxia no hepcidin regulation. European Journal of Applied Physiology, 120(6), 1331-1340. doi:10. 1007/s00421-020-04365-X

Hennigar, S.R., McClung, J.P., \& Pasiakos, S.M. (2017). Nutritional interventions and the IL-6 response to exercise. The FASEB Journal, 31(9), 3719-3728. PubMed ID: 28507168 doi:10.1096/fj.201700080R

Jennrich, R.I., \& Schluchter, M.D. (1986). Unbalanced repeated-measures models with structured covariance matrices. Biometrics, (4), 805-820. doi: $10.2307 / 2530695$

Jeukendrup, A.E. (2017). Training the gut for athletes. Sports Medicine, 47(S1), 101-110. doi:10.1007/s40279-017-0690-6

Karl, J.P., Lieberman, H.R., Cable, S.J., Williams, K.W., Young, A.J., \& McClung, J.P. (2010). Randomized, double-blind, placebo-controlled trial of an iron-fortified food product in female soldiers during military training: Relations between iron status, serum hepcidin, and inflammation. The American Journal of Clinical Nutrition, 92(1), 93-100. PubMed ID: 20444958 doi:10.3945/ajen.2010.29185

Kroot, J.J., Laarakkers, C.M., Geurts-Moespot, A.J., Grebenchtchikov, N., Pickkers, P., van Ede, A.E., ... Sweep, F.C. (2010). Immunochemical and mass-spectrometry-based serum hepcidin assays for iron metabolism disorders. Clinical Chemistry, 56(10), 1570-1579. PubMed ID: 20739637 doi:10.1373/clinchem.2010.149187

Leverve, X., Batandie, C., \& Fontaine, E. (2007). Choosing the right substrate. Sepsis-New insights, new therapies. Novartis Foundation Symposium 280:108-121. PubMed ID: 17380791

Margolskee, R.F., Dyer, J., Kokrashvili, Z., Salmon, K.S., Ilegems, E., Daly, K., ... Shirazi-Beechey, S.P. (2007). T1R3 and gustducin in gut sense sugars to regulate expression of Na+-glucose cotransporter 1. Proceedings of the National Academy of Sciences, 104(38), 1507515080. doi:10.1073/pnas.0706678104

McKay, A.K., Peeling, P., Pyne, D.B., Welvaert, M., Tee, N., Leckey, J.J., ... van Swelm, R.P. (2019b). Acute carbohydrate ingestion does not influence the post-exercise iron-regulatory response in elite ketoadapted race walkers. Journal of Science and Medicine in Sport, 22(6), 635-640. doi:10.1016/j.jsams.2018.12.015

McKay, A.K., Peeling, P., Pyne, D.B., Welvaert, M., Tee, N., Leckey, J.J., .. Swinkels, D.W. (2019a). Chronic adherence to a ketogenic diet modifies iron metabolism in elite athletes. Medicine \& Science in Sports \& Exercise, 51(3), 548-555. doi:10.1249/MSS.0000000 000001816

Nemeth, E., Rivera, S., Gabayan, V., Keller, C., Taudorf, S., Pedersen, B.K., \& Ganz, T. (2004a). IL-6 mediates hypoferremia of inflammation by inducing the synthesis of the iron regulatory hormone hepcidin. The Journal of Clinical Investigation, 113(9), 12711276. doi:10.1172/JCI200420945

Nemeth, E., Tuttle, M.S., Powelson, J., Vaughn, M.B., Donovan, A., Ward, D.M., ... Kaplan, J. (2004b). Hepcidin regulates cellular iron efflux by binding to ferroportin and inducing its internalization. Science, 306(5704), 2090-2093. doi:10.1126/science.1104742

Pedersen, B.K., \& Fischer, C.P. (2007). Beneficial health effects of exercise-the role of IL-6 as a myokine. Trends in Pharmacological Sciences, 28(4), 152-156. PubMed ID: 17331593 doi:10.1016/j.tips. 2007.02.002

Peeling, P., Dawson, B., Goodman, C., Landers, G., Wiegerinck, E.T., Swinkels, D.W., \& Trinder, D. (2009). Effects of exercise on hepcidin response and iron metabolism during recovery. International Journal of Sport Nutrition and Exercise Metabolism, 19(6), 583-597. PubMed ID: 20175428 doi:10.1123/ijsnem.19.6.583

Peeling, P., McKay, A.K., Pyne, D.B., Guelfi, K.J., McCormick, R.H., Laarakkers, C.M., . . Burke, L.M. (2017). Factors influencing the 
post-exercise hepcidin-25 response in elite athletes. European Journal of Applied Physiology, 117(6), 1233-1239. PubMed ID: 28409396 doi:10.1007/s00421-017-3611-3

Peeling, P., Sim, M., Badenhorst, C.E., Dawson, B., Govus, A.D., Abbiss, C.R., ... Trinder, D. (2014). Iron status and the acute post-exercise hepcidin response in athletes. PloS One, 9(3), e93002. PubMed ID: 24667393 doi:10.1371/journal.pone.0093002

R Core Team. (2017). R: A language and environment for statistical computing. Vienna, Austria: R Foundation for Statistical Computing; 2014. Retrieved from https://www.R-project.org/

Rowland, T. (2012). Iron deficiency in athletes: an update. American Journal of Lifestyle Medicine, 6(4), 319-327. doi:10.1177/15598276 11431541

Sim, M., Garvican-Lewis, L.A., Cox, G.R., Govus, A., McKay, A.K.A., Stellingwerff, T., Peeling, P. (2019). Iron considerations for the athlete: A narrative review. European Journal of Applied Physiology, 119(7), 1463-1478. PubMed ID: 31055680 doi:10.1007/s00421-019-04157-y

Starkie, R., Hargreaves, M., Rolland, J., \& Febbraio, M. (2005). Heat stress, cytokines, and the immune response to exercise. Brain, Behavior, and Immunity, 19(5), 404-412. PubMed ID: 16061150 doi:10.1016/j.bbi.2005.03.005
Steensberg, A., Febbraio, M.A., Osada, T., Schjerling, P., Hall, G., Saltin, B., \& Pedersen, B.K. (2001). Interleukin-6 production in contracting human skeletal muscle is influenced by pre-exercise muscle glycogen content. The Journal of Physiology, 537(2), 633-639. doi:10.1111/j. 1469-7793.2001.00633.x

Swinkels, D.W., Girelli, D., Laarakkers, C., Kroot, J., Campostrini, N., Kemna, E.H., \& Tjalsma, H. (2008). Advances in quantitative hepcidin measurements by time-of-flight mass spectrometry. PLoS One, 3(7), e2706. PubMed ID: 18628991 doi:10.1371/journal.pone. 0002706

Thomas, D.T., Erdman, K.A., \& Burke, L.M. (2016). Position of the academy of nutrition and dietetics, dietitians of Canada, and the American college of sports medicine: Nutrition and athletic performance. Journal of the Academy of Nutrition and Dietetics, 116(3), 501-528. PubMed ID: 26920240 doi:10.1016/j.jand.2015. 12.006

Trumbo, P., Yates, A.A., Schlicker, S., \& Poos, M. (2001). Dietary reference intakes: Vitamin A, vitamin $\mathrm{K}$, arsenic, boron, chromium, copper, iodine, iron, manganese, molybdenum, nickel, silicon, vanadium, and zinc. Journal of the American Dietetic Association, 101(3), 294-301. doi:10.1016/S0002-8223(01)00078-5 